\title{
Selection and antitumor activity of anti-Bcl-2 DNAzymes
}

\author{
Xinhui Yang ${ }^{1}$, Zhi Li ${ }^{1,2}$, Lu Zhang ${ }^{1,2}$, Jiang $\mathrm{He}^{1,2}$ and Lun-Quan Sun ${ }^{1,2 *}$
}

1. Center for Molecular Medicine, Xiangya Hospital; Collaborative Innovation Center for Cancer Medicine, Central South University, Changsha, 410078, China

2. Key Laboratory of Molecular Radiation Oncology, Hunan Province, China

* Correspondence to:

Prof. Lun-Quan Sun

Xiangya Hospital, Central South University

87 Xiangya Road, Changsha 410078, China

Tel: +8673184327646

Fax: +86 73184327695

Email: lunquansun@csu.edu.cn 


\begin{abstract}
Apoptosis pathway has become one of the important targets for therapeutic exploration for cancer therapy. The increased Bcl-2 protein level and phosphorylation is implicated in a decreased chemotherapeutic response in many cancers. BCL-2 inhibitors have been developed as direct inducers of apoptosis. However, resistance to BCL2 inhibitors has been emerging and thus considerable effort has been made to seek novel approaches to BCL2 suppression. In this report we describe an in vitro DNAzyme selection strategy resulting in molecules that are effective in suppressing expression of the target gene BCL-2 in vitro. A 3'inverted modification was shown to significantly increase the DNAzyme stability in serum and the modified DNAzyme delivered by an osmotic pump chemosensitized human prostate cancer to Taxol in vivo. Thus this study provides an alternative strategy for potential BCL-2-targetd therapy.
\end{abstract}

Key Words: DNAzyme; Bcl-2; Apoptosis; Chemoresistance 


\section{INTRODUCTION}

RNAs with their predictable affinity for complementary sequences are appealing targets for exogenous regulation of gene expression. Taking advantages of hybridization as a means for gene suppression is mostly related to the advance of single stranded oligodeoxynucleotides (ODNs) and double stranded small interfering RNA (siRNA) based approaches [1]. Other nucleic acids-based-technologies with unique properties and attributes may also find potentials in therapeutic applications. RNA-cleaving catalytic DNA (DNAzyme) has been one of the promising strategies for such applications [2]. DNAzymes interact with targets by hybridization and catalyze their cleavage/destruction of the target RNAs without requiring any host-encoded proteins. The nuclease independent activity of catalytic DNA or DNAzymes provides some opportunities that are currently beyond the scope of RNAi pathway-dependent siRNA and ribonuclease $\mathrm{H}$ dependent ODNs. With known targets, one could design corresponding DNAzymes to down-regulate the disease target genes. To date, DNAzymes, mainly "10-23" type [3], have been extensively explored for their ability to suppress the genes related to a number of disorders, including cancers [4-7]. Recent successes in clinical trials of the DNAzymes in squamous cell cancer and nasopharyngeal carcinoma have renewed the interests in development of DNAzymes as anti-cancer therapeutics $[8,9]$.

Apoptosis is a key pathway mediating cell death and is crucial for normal development and homeostasis. The BCL-2 family proteins and the inhibitors of apoptosis proteins (AIPs) are the major regulators of the apoptotic process, whereas caspases, a family of cysteine proteases, are the major executioners of this process [10]. The mechanisms by which BCL-2 family proteins regulate cell death are largely unknown, 
though it is thought that their function depends mostly on their ability to modulate the release of proteins from the inter-membrane space of the mitochondria. The BCL-2 family includes both pro- and anti-apoptosis proteins, among which BCL-2 protein inhibits the release of cytochrome $\mathrm{C}$ from the mitochondria, preventing the activation of caspase-9 and downstream caspases that lead to apoptosis [11].

Elevated Bcl-2 protein expression is evident in many tumors and the increased Bcl2 protein level and phosphorylation is implicated in a decreased chemotherapeutic response seen in many patients [12]. For these reasons the apoptosis pathway has become one of the important targets for therapeutic exploration for cancer therapy. Downregulating Bcl-2 with antisense and ribozyme molecules leads to an induction of apoptosis in many tumor cell lines. In vivo treatment of mice with the BCL-2 targeted antisense can delay the emergence androgen independent prostate cancer [13] and chemosensitize tumor cells to cancer treatment [14]. BCL2 inhibitors have been developed as direct inducers of apoptosis. ABT-199 (venetoclax) received breakthrough therapy designation from the FDA due to its apparent efficacy in CLL and AML [15]. However, resistance to BCL2 inhibitors has been emerging and thus considerable effort has been expended seeking novel approaches to BCL2 proteins [16].

In this report we describe an in vitro DNAzyme selection strategy resulting in molecules that are effective in suppressing expression of the target protein $\mathrm{Bcl}-2$ both in vitro and in vivo, thus provide an alternative strategy for potential BCL-2-targetd therapy.

\section{MATERIALS AND METHODS}

\subsection{Cell Lines}


Prostate cancer cell line DU145 was maintained in RPMI-1640 media (Gibco, Life Technologies). PC3 prostate cancer cells were maintained in DMEM media. All media was supplemented with $10 \%$ fetal bovine serum, $2 \mathrm{mM} \mathrm{L-glutamine,} 50 \mathrm{U} / \mathrm{ml}$ penicillin (Gibco Life Technologies) and $50 \mu \mathrm{g} / \mathrm{ml}$ of streptomycin (Gibco Life Technologies).

\subsection{DNAzymes and Oligonucleotides}

Both unmodified and modified DNAzymes used in in vitro screening, and cellular and in vivo activity experiments were supplied by Oligos Etc (Portland, OR). All DNAzymes contained a "10-23" catalytic motif (ggctagctacaacga) and 7- 9 bp hybridizing arms specific for RU cleavage sites in a $3 \mathrm{~kb}$ of the Bcl-2 transcript. Primer extension was performed in multiplex selection reactions with the primers as follows: 5'cacagcattaaacattgaacag-3'; 5'-tggaacttttttttgtcagg-3'; 5'-tcctcacgttcccagccttc-3' ; 5'cagacattcggagaccacac-3' ; 5'-cagtattgggagttgggggg-3'; 5'- ccaactcttttcctcccacc-3' ; 5'cgacgttttgcctgaagactg-3'; 5'- cagggccaaactgagcagag-3'; 5'- atcctcccccagttcacccc-3'; 5'ggatgcggctgtatgggg-3'; 5'-aggccacgtaaagcaactctc-3'.

\subsection{Multiplex in vitro selection}

Linear DNA needed for the production of a template for transcription was produced by digesting Bcl-2 plasmid with $K p n I$ (New England Biolabs) for 2 hours at $37^{\circ} \mathrm{C}$ in the supplied buffer. Unlabeled RNA was transcribed from the T7 promoter of $1 \mu \mathrm{g}$ of linearized Bcl-2 plasmid using T7 RNA polymerase at $37^{\circ} \mathrm{C}$ for $3 \mathrm{~h}$ using an RNA transcription kit (Epicentre Technology, Madison, WI). 
Multiple DNAzymes ( $5 \mathrm{nM}, 50 \mathrm{nM}$ and $500 \mathrm{nM})$ and Bcl-2 transcript $(2 \mu \mathrm{g})$ were preequilibrated at $85^{\circ} \mathrm{C}$ for $3 \mathrm{~min}$ in equal volumes of $50 \mathrm{mM}$ Tris- $\mathrm{HCl}, \mathrm{pH} 7.5,10 \mathrm{mM}$ $\mathrm{MgCl}_{2}, 150 \mathrm{mM} \mathrm{NaCl}$, and $0.01 \%$ SDS. The reaction was initiated by mixing the DNAzyme and the substrate together and incubating for $1 \mathrm{~h}$ at $37^{\circ} \mathrm{C}$. The reaction was stopped by precipitating in ethanol.

In order to detect cleavage points, primer extension of a reverse primer was employed with the primers described above. The 5 '-terminal of each primer $(1 \mu \mathrm{M})$ was radiolabeled in $60 \mathrm{mM}$ Tris- $\mathrm{HCl}, \mathrm{pH} 7.5,9 \mathrm{mM} \mathrm{MgCl}, 10 \mathrm{mM}$ dithiothreitol, $1 \mathrm{U}$ polynucleotide kinase (New England Biolabs, Beverly, MA) and $10 \mu \mathrm{Ci}$ of $\left[\gamma_{-}{ }^{32} \mathrm{P}\right] \mathrm{ATP}$ (Perkin Elmer) at $37^{\circ} \mathrm{C}$ for $45 \mathrm{~min}$ and $75^{\circ} \mathrm{C}$ for $10 \mathrm{~min}$. Primer extension was performed with 2 pmol of labeled primer and $400 \mathrm{ng}$ of DNAzyme cleaved RNA denatured at $70^{\circ} \mathrm{C}$ for $10 \mathrm{~min}$. The primer and template were allowed to anneal by cooling the reaction to room temperature before adding the first strand buffer, 200U of SuperScriptII reverse transcriptase, dithiothreitol and deoxyribonucleotides according to the manufacturer's instructions. This mixture was incubated for $25^{\circ} \mathrm{C}$ for $10 \mathrm{~min}, 42^{\circ} \mathrm{C}$ for $50 \mathrm{~min}, 70^{\circ} \mathrm{C}$ for $15 \mathrm{~min}$ and then stored at $4^{\circ} \mathrm{C}$ before analysis.

To determine the sequence at which the DNAzymes cleaved, sequencing reactions were performed on the double stranded Bcl-2 plasmid with the same $\gamma_{-}{ }^{32} \mathrm{P}$-labeled reverse primers as in the reverse extension reactions. These reactions were subject to linear amplification for 25 cycles at $95^{\circ} \mathrm{C}$ for $30 \mathrm{~s}, 60^{\circ} \mathrm{C}$ for $90 \mathrm{~s}$, and $72^{\circ} \mathrm{C}$ for $60 \mathrm{~s}$ (Murray, 1989). Primer extension or sequencing reactions were combined with equal volumes of stop buffer (Formamide, EDTA loading dye) before electrophoresis on a $6 \%$ denaturing polyacrylamide gel. The corresponding gel image was analyzed and band intensity 
determined with PhosporImager and ImageQuant software (Molecular Dynamics, Sunnyvale, CA).

\subsection{DNAzyme Kinetic Analysis}

The single turnover kinetics for the selected DNAzymes was performed using in vitro transcribed short substrate RNAs. Briefly, the primers with a T7 promoter sequence and the corresponding paired primers were used to generate the transcription templates. RNA substrates were transcribed from the templates with ${ }^{32} \mathrm{P}-\mathrm{rUTP}$ using the Epicenter In vitro Transcription kit using T7 RNA polymerase according to the manufacturer's instructions. Transcripts were run on a $16 \%$ polyacrylamide denaturing gel and transcripts of the correct size (approx $23 \mathrm{bp}$ ) were cut out of the gel and eluted overnight in $3 \mathrm{M}$ sodium acetate and precipitated in ethanol the following day.

To carry out the single turnover kinetics, excess DNAzyme $(10 \mu \mathrm{M})$ was used to cleave individual DNAzyme substrates at intervals of 5, 10, 20, 30 and 60 minutes in a buffer containing $50 \mathrm{mM}$, Tris- $\mathrm{HCl}, \mathrm{pH} 7.5$, and $20 \mathrm{mM} \mathrm{MgCl}$. Substrate and cleavage products were run on a $16 \%$ denaturing polyacrylamide gel. The progress of these reactions was determined by densitometry and analyzed by comparing the percentage of products produced against time. A curve was generated for the data (least-squares) using The equation $\% \mathrm{P}=\% \mathrm{P}_{\infty}-\mathrm{C} \cdot \exp [-\mathrm{kt}]$ was used for the first order rate constant $\left(\mathrm{k}_{\mathrm{obs}}\right)$, where $\% \mathrm{P}$ is the percentage product, $\% \mathrm{P}_{\infty}$ is the percentage product at $\mathrm{t}=\infty, \mathrm{C}$ is the difference in $\% \mathrm{P}$ between $\mathrm{t}=\infty$ and $\mathrm{t}=0$, and $\mathrm{k}$ is the first order rate constant [17].

\subsection{Transfection of tumor cell line with anti-Bcl-2 DNAzymes}


Prostate cancer cells (PC3 and DU145) were seeded in $60 \mathrm{~mm}^{2}$ dishes $\left(8 \times 10^{5} / \mathrm{dish}\right)$ to allow a confluence between $60 \%-80 \%$ was reached at the following day. DNAzymes were transfected with tetra meso (4-methylpyridyl) porphyrin (TMP) at a charge ratio of 3 [18]. The final concentration of DNAzyme in the transfection mix was $2 \mu \mathrm{M}$. Protein was extracted from the cells approximately 24 hours after transfection. Western blot analyses were performed for the DNAzyme effect on BCL-2 expression using the antibodies of anti-Bcl-2 mouse monoclonal antibody (1 $\mu \mathrm{g} / \mathrm{ml})$ (Santa Cruz), antiCytochrome $\mathrm{C}$ rabbit polyclonal antibody (Santa Cruz) and anti- $\beta$-actin monoclonal antibody (AC-74, Sigma). Binding of primary antibodies was detected with goat-antimouse (1/2000 dilution) or goat anti-rabbit (1/2000) (Santa Cruz Biotechnologies) horse radish peroxidase conjugated secondary antibodies. This binding was visualized with the ECL western blotting detection reagents.

\subsection{Cell Survival Assay}

PC3 and DU145 cells were seeded in 24 well plates so that the following day their density was between $60-80 \%$. Cells were then treated with $2 \mu \mathrm{M}$ DNAzyme complexed with TMP at a charge ratio of 3 for $4 \mathrm{~h}$ at $37^{\circ} \mathrm{C}$. The transfection complex was then removed and the cells cultured in complete media for $24 \mathrm{~h}$ followed by a MTS assay to determine the cell viability.

\subsection{Real Time RT-PCR Analysis}

PC3 cells treated with $2 \mu \mathrm{M}$ anti-Bcl-2 DNAzyme complexed with TMP at a charge ration of 3. RNA was extracted $20 \mathrm{~h}$ post transfection using TRIzol (Invitrogen) 
according to the manufacturer's instructions. Ten nanograms of total RNA was reverse transcribed and subjected to the following thermocycling conditions on an ABI7700 thermocycler: $48^{\circ} \mathrm{C}$ for $30 \mathrm{~min}, 95^{\circ} \mathrm{C}$ for $10 \mathrm{~min}$ and 40 cycles of $95^{\circ} \mathrm{C}$ for $15 \mathrm{~s}, 60^{\circ} \mathrm{C} 60 \mathrm{~s}$. Results were analyzed using the Sequence Detector Systems v1.7 software.

\subsection{In vivo testing of BCL-2 DNAzyme}

The in vivo mouse experiments were approved by the Animal Ethic Committee of St Vincent's Hospital, University of New South Wales, Sydney. Four groups of animal (5 mice each group) were used for anti-BCL-2 DNAzyme chemosensitization of human prostate cancer cells in xenograft mouse model (Saline, Taxol, DNAzyme and DNAzyme

+ Taxol). Human prostate cancer PC3 cells $\left(5 \times 10^{5} / 100 \mu l\right)$ were injected subcutaneously into the right hind leg of BALB/c nude mice (4-6 week old female). Palpable tumors ( $100 \mathrm{~mm} 3$, calculated using length $\mathrm{x}$ width $\mathrm{x}$ height $\mathrm{x} \pi / 6)$ grew within two weeks of implantation at which time the BCL-2 DNAzyme treatment started. DNAzyme DT912 was delivered using an osmotic pump and Taxol was administrated via i.p. route weekly. Tumor size was measured at the time points indicated.

\subsection{Statistical analysis}

All of the data were presented as means \pm SEM, and a P value less than 0.05 was considered to be statistically significant $(* \mathrm{p}<0.05)$.

\section{RESULTS}

3.1 Target selection within Bcl-2 mRNA 
One of the key issues for nucleic acids-based therapeutics is how to find the target sites within a given mRNA. To identify the accessible sites of 10-23 DNAzyme in Bcl-2 mRNA (Fig. 1A), we established a scheme for this purpose (Fig. 1B). We scanned the mRNA corresponding to the bcl-2 gene clone and found 141 potential AU and GU cleavage sites, which were further subjected to two thermodynamic analyses. The first analysis was on the thermodynamic stability of the enzyme-substrate heteroduplex as predicted by the hybridization free energy [19]. DNAzymes with the greatest heteroduplex stability indicated by a low free energy of hybridization (calculated using the nearest neighbor method), was often found to have the greatest kinetic activity. The second analysis was to examine if the arms of the DNAzyme had a high hairpin melting temperature (Tm), thus to avoid any intramolecular bonds. After completion of these analyses, 55 DNAzymes were designed and synthesized for in vitro multiplex selection.

In order to efficiently select active DNAzymes, in vitro selection was performed using a multiplex method, which enables a pool of DNAzymes to be screened for their ability to access and cleave RNA substrate under simulated physiological conditions. By using the sequence ladders as a guide to attribute cleavage bands to specific DNAzymes, the relative cleavage strength of each DNAzyme was determined by intensity of the cleavage products. DNAzymes were ranked according to their cleavage ability at lowest concentration $(5 \mathrm{nM})$. As a result, top five DNAzymes were chosen for further kinetic analyses. Figures 1C showed one of the representative gels for the BCL-2 DNAzyme selection.

3.2 Kinetic analysis of the selected DNAzymes against Bcl-2 
To characterize the selected DNAzymes biochemically, we performed kinetic analyses for their cleavage efficiency (Fig. 2A). Under single turnover conditions, DNAzymes DT899 and DT912 demonstrated very good substrate cleavage (75.3\% and $84.4 \%$ ) and kinetic activity with Kobs of $0.34 \mathrm{~min}^{-1}$ and $0.22 \mathrm{~min}^{-1}$ respectively. DT895 also had good kinetic activity $\left(0.31 \mathrm{~min}^{-1}\right)$, however the DNAzyme was only able to cleave $37.6 \%$ of its substrate (Fig. 2B). Considering the presence of a G-quartet in DT899, which could potentially cause non-specificity [20], DT912 was chosen for further cell and in vivo experiments.

3.3 Modification, stability and gene suppression activity of anti-BCL-2 DNAzymes To increase the resistance to nuclease degradation of DNAzymes in vivo, we carried out several types of chemical modifications for DT912. These modifications included four phosphorothioate linkages at both ends, 3'-inverted-T and four 2'-O-methyl at both ends (Fig. 3A). The modified DNAzymes were incubated with human serum up to $24 \mathrm{~h}$ at $37{ }^{\circ} \mathrm{C}$ and sampled at the designated time points. The modified DNAzymes were extracted, labelled with $\gamma-{ }^{32}$ P-ATP and assayed on $16 \%$ PAGE. The intact DNAzyme intensity was quantitated by densitometry. As shown in Fig. 3B, the 3'-inverted modification significantly increased DT912 stability in human serum, in comparison with other modifications. This indicated that the DNAzyme oligonucleotide might be predominantly attacked by the 3 '-exonucleases in serum.

To assess anti-BCL-2 DNAzymes for their ability to down regulate the target gene expression in cells, PC3 cells were transfected with the modified DT912 and DT910, and assayed by western blots for BCL-2 expression. Both DNAzymes could effectively 
suppress the BCL-2 expression, compared with the control (Fig. 3C). To investigate if the anti-BCL-2 DNAzymes impacted on the downstream molecular events in apoptosis, the cytochrome C (Cyto C) level was also examined. As expected, down-regulation of BCL2 by the DNAzymes caused marked increase of the Cyto C release (Fig. 3C). The DNAzyme effect on BCL-2 expression was further confirmed by RT-PCR at the transcriptional level (Fig. 3D).

\subsection{Effect of DT912 on cell survival}

Following the test of the DNAzymes in Western and RT-PCR assays, DT912 was further examined for tits effect on cell survival as an indication of apoptotic response. In the assay, two prostate cancer cell lines were used, of which BCL-2 was highly expressed in PC3 cells while DU145 was BCL-2 poorly expressed. Treatment of PC3 cells with DT912 and the control (with randomized arms and inverted catalytic core sequence, based on DT912) significantly reduced the cell survival (Fig. 4A). In contrast, there was only marginal effect of DT912 on DU145 cell survival (Fig. 4B), suggesting that the prostate cancer cells treated with anti-BCL-2 DNAzyme was provoked to undergo apoptosis in a target gene dependent manner.

3.5 In vivo antitumor activity of anti-BCL-2 DNAzyme

In order to demonstrate that down-regulation of the bcl-2 gene resulted in chemosensitization of tumor cells to anticancer drugs, murine models with human PC3 prostate cancer xenograft was used to determine if the sensitivity to the chemotherapeutics was enhanced. In the experiments, Alzet osmotic pumps as a delivery 
vehicle for DNAzyme oligonucleotides in tumor bearing mice were surgically implanted in the peritoneum of the mouse via the abdominal route. The Alzet model1002 pump is a capsule shaped pump $(1.5 \times 0.6 \mathrm{~cm})$ and delivers a total volume of $0.5 \mathrm{ml}$ at a rate of 0.25 $\mu \mathrm{l} / \mathrm{h}$ over a period of 14 days. The pump was filled with a saline solution containing the DNAzyme oligonucleotide, which resulted in a dose rate of $12.5 \mathrm{mg} / \mathrm{kg} / \mathrm{d}$. Where indicated, some mice received $25 \mathrm{mg} / \mathrm{kg}$ Taxol by intraperitoneal route in a $200 \mu \mathrm{l}$ injection once weekly post-surgery for the duration of the study. As shown in Figures 4C, D, combination of DT912 and Taxol treatment markedly inhibited PC3 tumor growth, compared with the groups of the DNAzyme alone or Taxol alone. When the tumor tissues were harvested at the end of the tests, BCL-2 mRNA levels were shown to be markedly reduced in the DT912-treated mice, indicating that the chemosensitization of PC3 tumor was due to the suppression of BCL-2 expression by the anti-BCL-2 DNAzyme.

\section{DISCUSSION}

Distorted apoptosis pathways are well-accepted hallmark of cancer cells that contributes to tumor progression and to drug resistance [21]. Alterations in the activity of BCL-2 family members are frequently found in cancer cells and these contribute to an increased apoptotic threshold. Broad arrays of "OMICS" and functional data have provided a solid rationale to develop small-molecule BCL-2 inhibitors and other molecular

modalities that pharmacologically and genetically target cancer cells. Here, we show that targeting BCL-2 by a DNAzyme can sensitize human prostate cancer cells to chemotherapeutics by promoting the release of cytochrome $\mathrm{C}$ and lowering the apoptotic 
threshold. This work provides a novel alternative for BCL-2 targeted strategy for cancer therapy.

Relative to antisense oligonucleotide and RNA interference, DNAzyme has its unique features. DNAzymes exhibit greater substrate flexibility than other nucleic acidbased agents since their substrate binding domain could vary with the target sequence as long as the catalytic core is unchanged [22-24]. All these advantages make DNAzymes promising candidates for targeted therapy. However, as for all the nucleic acid-based agents, target selection and delivery are the keys for the successful use of them both biologically and clinically. In this study, we used a multiplex selection strategy, in which the accessible and cleavable sites for DNAzymes could be identified in a high-throughput and accurate biochemical manner with a consistence between in vitro and in vivo activities. Instead of using intratumoral injection as reported in the many studies, which may not be applicable in clinical settings, we here employed an osmotic pump as a delivery vehicle for DNAzymes via the abdominal route and demonstrated a viable means for efficient DNAzyme delivery.

Chemically, DNAzyme is comprised of deoxyoligonucleotides, which are more stable and easier to synthesize. Modification of DNAzymes could further enhance the stability in serum without affecting the catalytic activity [25, 26]. Although the modifications with phosphorothioate and 2'-O-methyl have been extensively utilized in DNA oligonucleotides, the issues in toxicity and efficiency with such modifications are still the major concerns for clinical application [27]. The 3'-inverted modifications, which can prevent the DNA or RNA from 3'-exonuclease degradation, do not introduce new chemistries into the molecules and maintain the modified DNA as natural entities. In 
the present study, we tested several different modification schemes and showed that the 3'inversion greatly increased the stability of the DNAzyme in serum while both in vitro and in vivo activities was not compromised with possibly low toxicity, which may warrant a pursuit for further preclinical and clinical testing of the 3 '-inverted anti-BCL-2 DNAzyme.

\section{ACKNOWLEGEMENT}

This work is partially supported by a Key R\&D grant from Ministry of Science and Technology China (2013BAI01B07), National Science Foundation China (81172188) and Specialized Research Fund for the Doctoral Program of Higher Education, Ministry of Education, China (20110162110010). Part of work was performed at formerly Johnson and Johnson Research Sydney. 


\section{REFERENCE}

1. L. Zhang, L. Yang, J.J. Li, L. Sun, Potential use of nucleic acid-based agents in the sensitization of nasopharyngeal carcinoma to radiotherapy. Cancer Lett. 323 (2012)110.

2. R. Bhindi, R.G. Fahmy, H.C. Lowe, et al., Brothers in arms: DNAzymes, siRNA and the emerging wave of small molecule nucleic acid-based gene silencing strategies, Am. J. Pathol. 171 (2007) 1079-88.

3. S.W. Santoro, G.F. Joyce, A general purpose RNA cleaving DNA enzyme, Proc. Natl. Acad. Sci. USA 94 (1997) 4262-6.

4. L.Q. Sun, M.J. Cairns, W.L. Gerlach, et al., Suppression of smooth muscle cell proliferation by a c-myc RNA cleaving deoxyribozyme, J. Biol. Chem. 274 (1999) 17236- 41.

5. G.S. Zhang, C.R. Dass, E. Sumithran E, et al., DNAzyme targeting c-jun inhibits microvascular endothelial cell proliferation, migration, microtubule formation, corneal neovascularization and malignant melanoma growth, J. Nat. Cancer Inst. 96 (2004) 683-96.

6. Z.X. Lu, X.Q. Ma, L.F. Yang, et al., DNAzymes targeted to EBV-encoded latent membrane protein-1 induce apoptosis and enhance radiosensitivity in nasopharyngeal carcinoma. Cancer Lett. 265 (2008) 226-38.

7. M. Cieslak, J. Niewiarowska, M. Nawrot, et al., DNAzymes to beta 1 and beta 3 mRNA down-regulate expression of the targeted integrins and inhibit endothelial cell capillary tube formation in fibrin and matrigel, J. Biol. Chem. 277 (2002) 6779-87.

8. E.A. Cho, F.J. Moloney, H. Cai, et al., Safety and tolerability of an intratumorally injected DNAzyme, Dz13, in patients with nodular basal-cell carcinoma: a phase 1 first-in-human trial (DISCOVER), Lancet 381 (2013) 1835-43.

9. Y. Cao, L.F. Yang, W.Z. Jiang, et al., Therapeutic Evaluation of Epstein-Barr VirusEncoded Latent Membrane Protein-1 Targeted DNAzyme for Treating of Nasopharyngeal Carcinomas, Molecular Therapy 22 (2013) 371-7.

10. P. Juin, O. Geneste, F. Gautier, S. Depil, M. Campone, Decoding and unlocking the BCL-2 dependency of cancer cells, Nature Rev. Cancer 13(2013) 455-465.

11. A. Gross, BCL-2 family proteins as regulators of mitochondria metabolism, Biochim. 
Biophys. Acta 1857 (2016) 1243-1246.

12. K.H. Khan, M. Blanco-Codesido, L.R. Molife, Cancer therapeutics: Targeting the apoptotic pathway. Crit Rev Oncol Hematol. 90(2014):200-19.

13. H. Miyake, A. Tolcher and M.E. Gleave, Antisense Bcl-2 oligonucleotides inhibit progression to androgen independence after castration in the shionogi tumor model. Cancer Res. 59(1999) 4030-4034.

14. S. Leung, H. Miyake, T. Zellweger, et al., Synergistic chemosensitization and inhibition of progression to androgen independence by antisense Bcl-2 oligonucleotide and paclitaxel in the LNCaP prostate tumor model, Int. J. Cancer 91(2001) 846-850.

15. A.J. Souers, J.D. Leverson, E.R. Boghaert, et al., ABT-199, a potent and selective BCL-2 inhibitor, achieves antitumor activity while sparing platelets, Nat Med. 19(2013) 202-8.

16. R.S. Soderquist and A. Eastman, Novel approaches to bcl2 inhibition BCL2 Inhibitors as Anticancer Drugs: A Plethora of Misleading BH3 Mimetics, Mol Cancer Ther., 15 (2016) 2011-17.

17. M.J. Cairns, A. King, L.Q. Sun, Optimization of the 10-23 DNAzyme-substrate pairing interactions enhanced RNA cleavage activity at purine-cytosine target sites, Nucleic Acids Research, 31 (2003) 2883-2889.

18. L. Benimetskaya, G.B. Takle, M. Vilenchik, et al., Cationic porphyrins: novel delivery vehicles for antisense oligodeoxynucleotides. Nucleic Acids Res. 26(1998)5310-7.

19. N. Sugimoto, S. Nakano, M. Katoh, et al., Thermodynamic parameters to predict stability of RNA/DNA hybrid duplexes. Biochemistry 34(1995) 11211-6.

20. S. Agrawal, P.L. Iadarola, J. Temsamani, et al., Effect of G-rich sequences on the synthesis, purification, hybridization, cell uptake, and hemolytic activity of oligonucleotides, Bioorg. Med. Chem. Lett. 6(1996) 2219-24.

21. D. Hanahan, R. A. Weinberg, Hallmarks of cancer: the next generation, Cell 144(2011)646-74.

22. J. Goodchild, Hammerhead ribozymes: Biochemical and chemical considerations. Current opinion in molecular therapeutics 2(2000) 272-281. 
23. T. Shimayama, S. Nishikawa, K. Taira, Generality of the nux rule: Kinetic analysis of the results of systematic mutations in the trinucleotide at the cleavage site of hammerhead ribozymes. Biochemistry 34(1995) 3649-3654.

24. T. Kuwabara, M. Warashina, T. Tanabe, et al., Comparison of the specificities and catalytic activities of hammerhead ribozymes and DNA enzymes with respect to the cleavage of bcr-abl chimeric 16 (b2a2) mrna. Nucleic acids research 25(1997)30743081.

25. E. Finkel, DNA cuts its teeth--as an enzyme. Science 286(1999) 2441-2442.

26. M. Sioud, M. Leirdal, Design of nuclease resistant protein kinase calpha DNA enzymes with potential therapeutic application. Journal of molecular biology 296(2000) 937-947.

27. J.A. Engelhardt, Comparative Renal Toxicopathology of Antisense Oligonucleotides. Nucleic Acid. Ther. 26(2016)199-209. 


\section{FIGURE LEGENDS}

Figure 1 Anti-BCL-2 DNAzyme selection scheme. A, "10-23" model of DNAzyme and its target. B, Streamline of multiplex selection strategy. C, A representative gel derived from the multiplex selection of anti-BCL-2 DNAzymes.

Figure 2 Biochemical analyses of the selected DNAzymes. A, Curves of DNAzyme cleavage efficiency with time. B, the kinetic parameters of the DNAzymes.

Figure 3 Stability and gene suppression of anti-BCL-2 DNAzymes. A, schematic description of DNAzyme modifications. B, Stability of the modified DT912 in human serum. C, Western blot analyses of DT912 in PC3 cells. D, RT-PCR analyses of DT912 for its activity of inhibiting BCL-2 mRNA expression.

Figure 4 In vitro and in vivo testing of DT912 in human prostate cancer. (A, B) PC3 (BCL-2 high) and DU145 (BCL-2 low) cells were transfected with DT912 and control and MTS assay was performed $24 \mathrm{~h}$ post transfection. C, PC3 cells $\left(5 \times 10^{5} / 100 \mu \mathrm{l}\right)$ were inoculated subcutaneously into BALB/c nude mice and grew for two week. BCL-2 DNAzyme treatment started at day 14 by sing an osmotic pump and Taxol was administrated via i.p. route weekly $(25 \mathrm{mg} / \mathrm{kg})$. Tumor size was measured at the time points indicated. D, Xenograft tissues were harvested at the end of experiment and subjected to RNA extraction. RT-PCR was performed to measure relative level of BCL-2 mRNA (ratio of BCL-2 and $\beta$-actin). The data were from three independent experiments and presented as mean \pm SEM. 
A Bcl-2 mRNA

3' NNNNNNN RNNNNNN5'

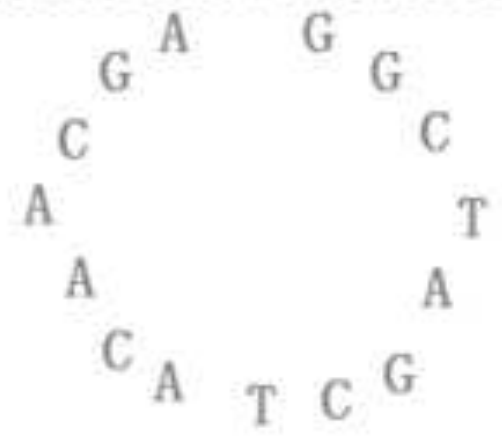

B

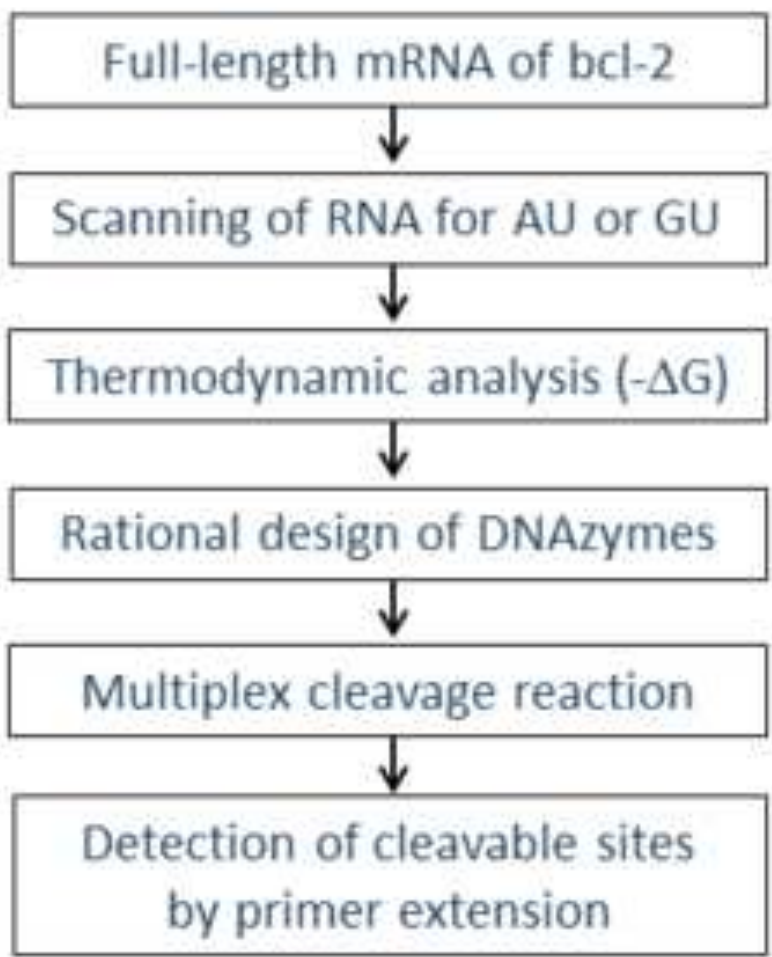

C

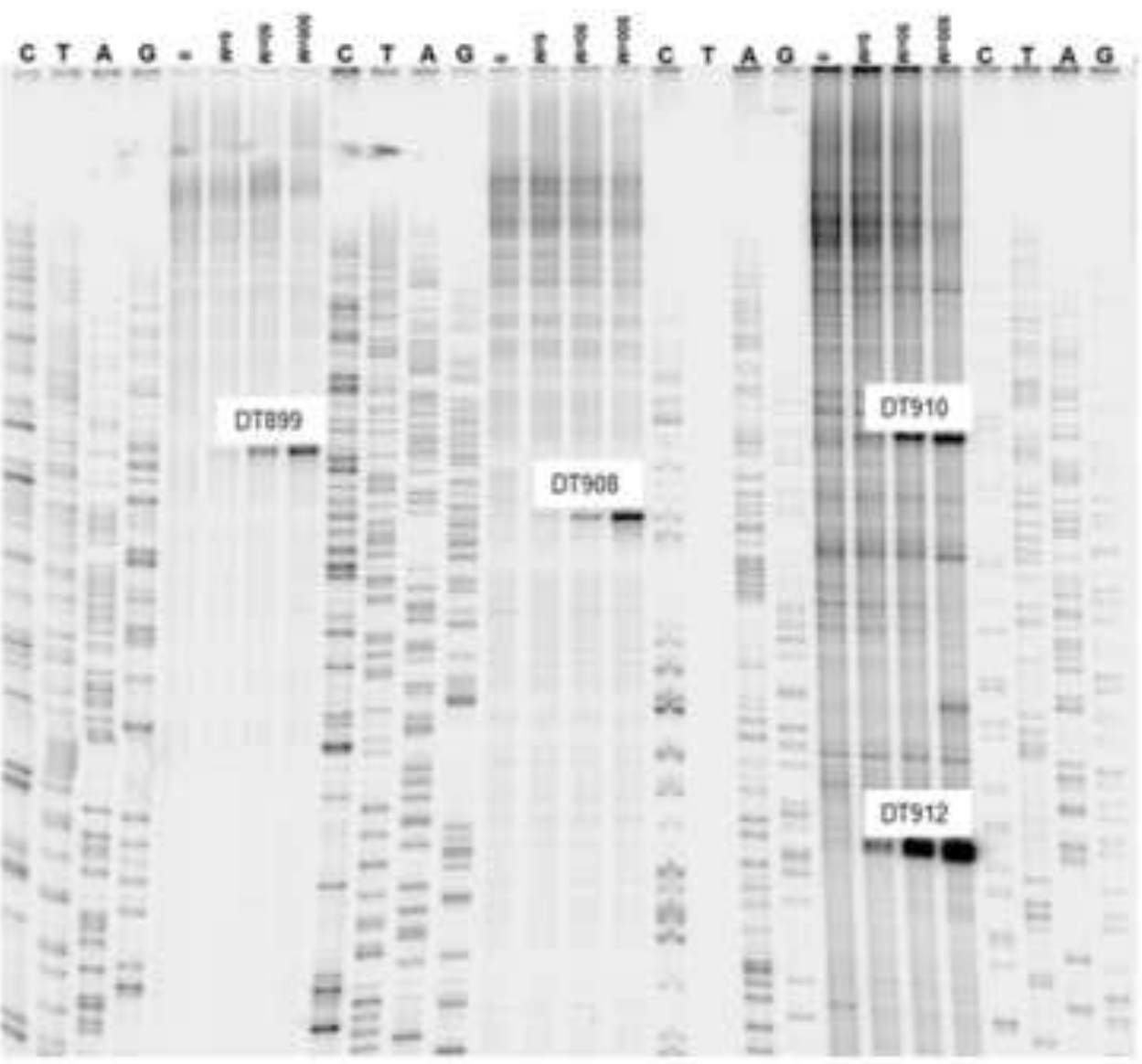

by primer extension 
A

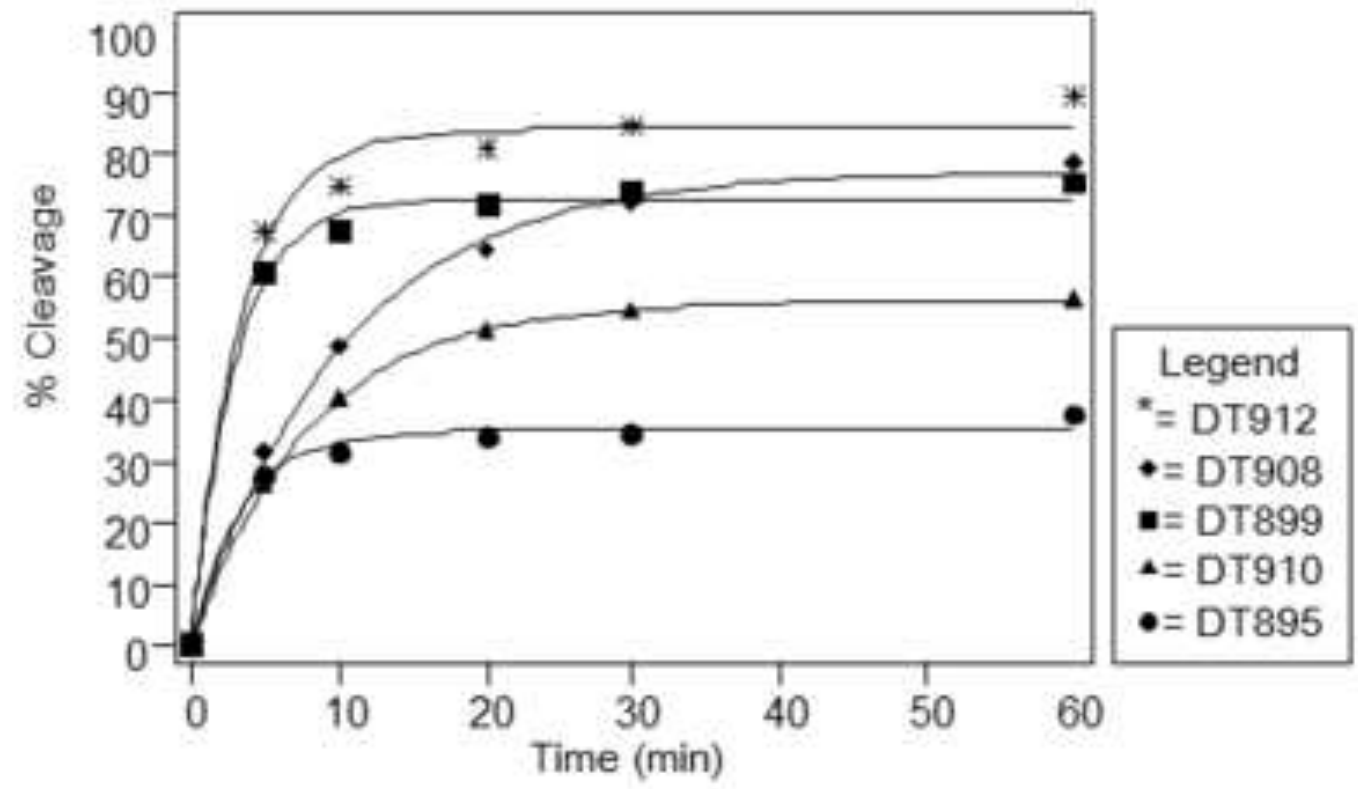

B

\begin{tabular}{|c|c|c|c|c|c|c|}
\hline \multicolumn{7}{|c|}{ Kinetic profiles of the selected DNAzymes targeting bel-2 } \\
\hline DNAzyme & Sequence & $\begin{array}{l}\text { In vitro } \\
\text { Activity }\end{array}$ & $\begin{array}{l}\text { Cleavage } \\
\text { site }\end{array}$ & $-\Delta G^{\circ}$ & $\begin{array}{l}\text { \% Substrate } \\
\text { Cleaved }\end{array}$ & $\begin{array}{l}K_{\text {obs }} \\
\left(\mathrm{min}^{-1}\right)\end{array}$ \\
\hline DT895 & cccagttcaggctagctacaacgacccgtccet & + & GU & 32.80 & 37.6 & 0.31 \\
\hline DT899 & ggggccgtaggctagctacaacgaagttccaca & + & GU & 28.8 & 75.3 & 0.34 \\
\hline DT908 & ttttcccaggctagctacaacgacgctgtcct & + & AU & 27.90 & 78.5 & 0.097 \\
\hline DT910 & ccctgttgaggctagctacaacgacatccctg8 & $H$ & AU & 28.40 & 56.3 & 0.12 \\
\hline DT912 & cacagccaaggctagctacaacgagtgccatgt & +H & GU & 26.7 & 84,4 & 0.22 \\
\hline
\end{tabular}


A.

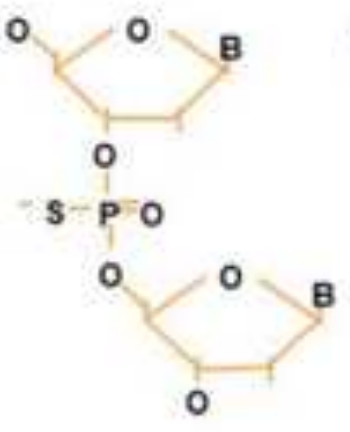

Phosphorothioate

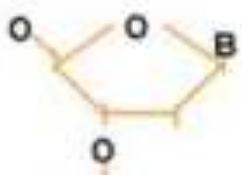

O-PO

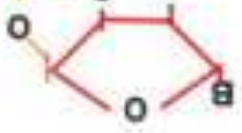

3'-inversion

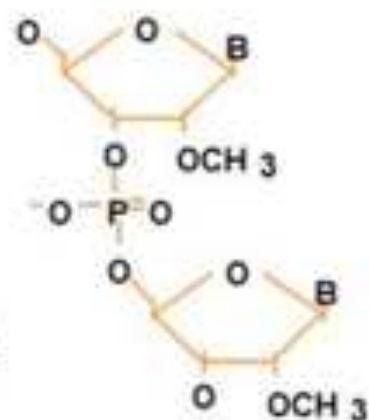

2'-0-methyl

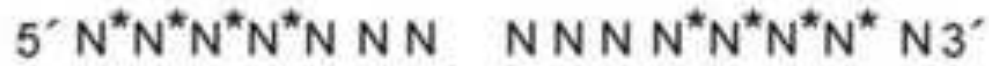

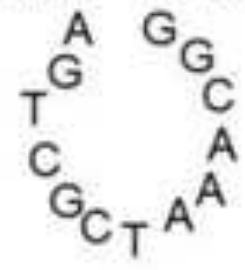

C

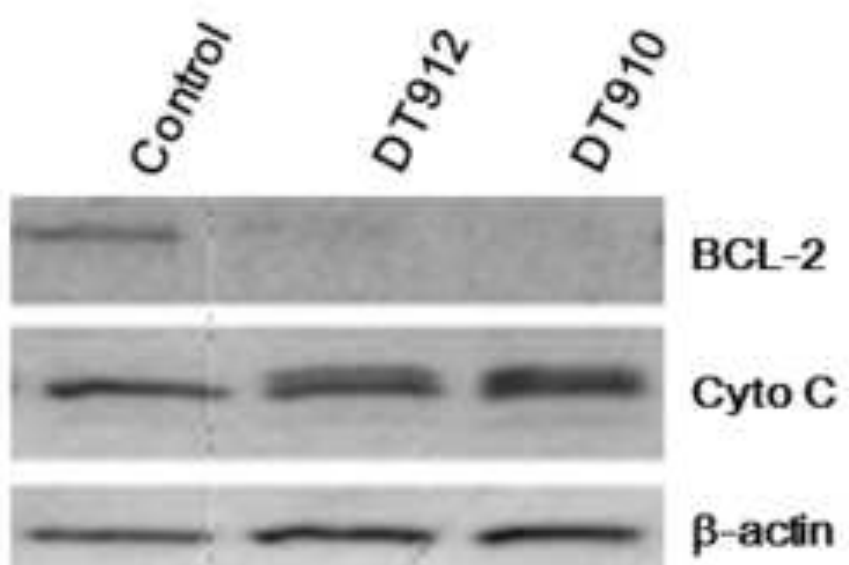

B

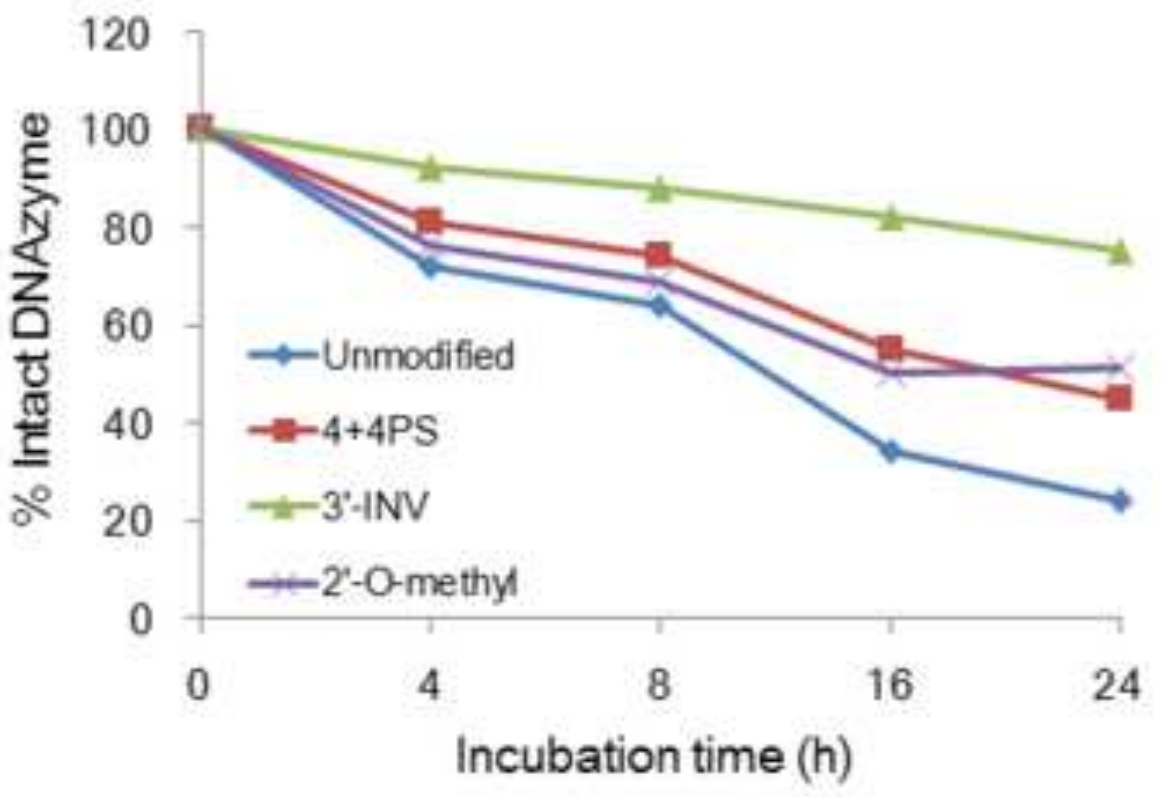

D

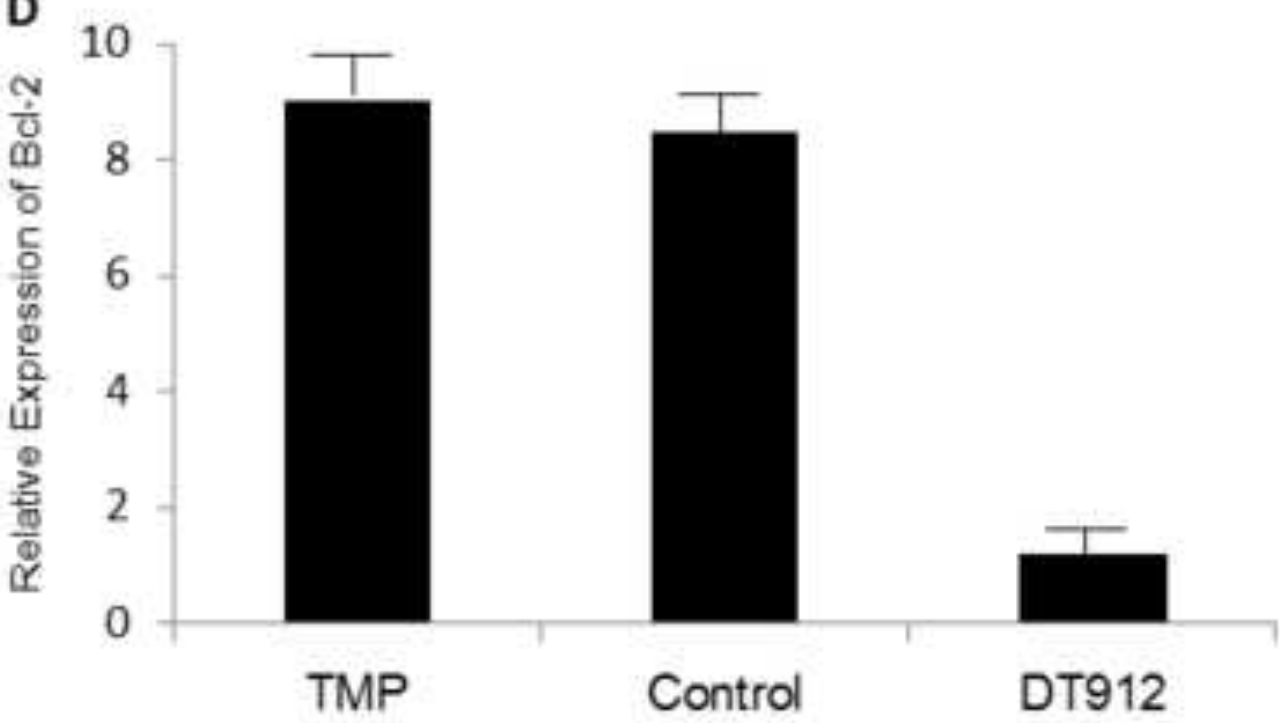




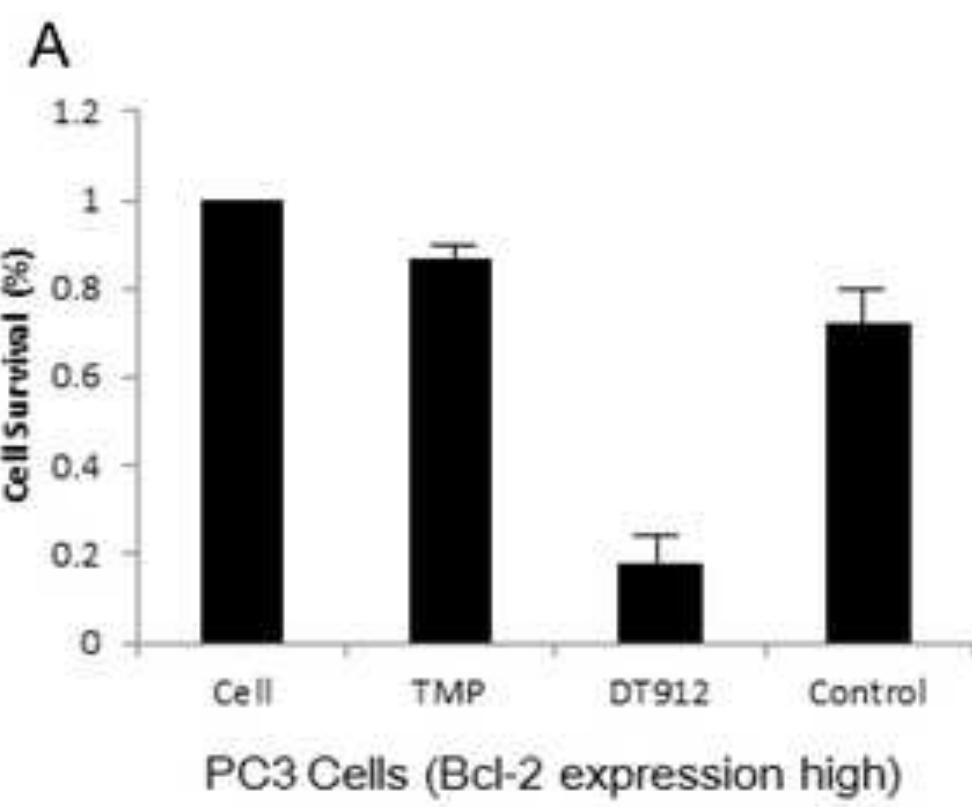

C

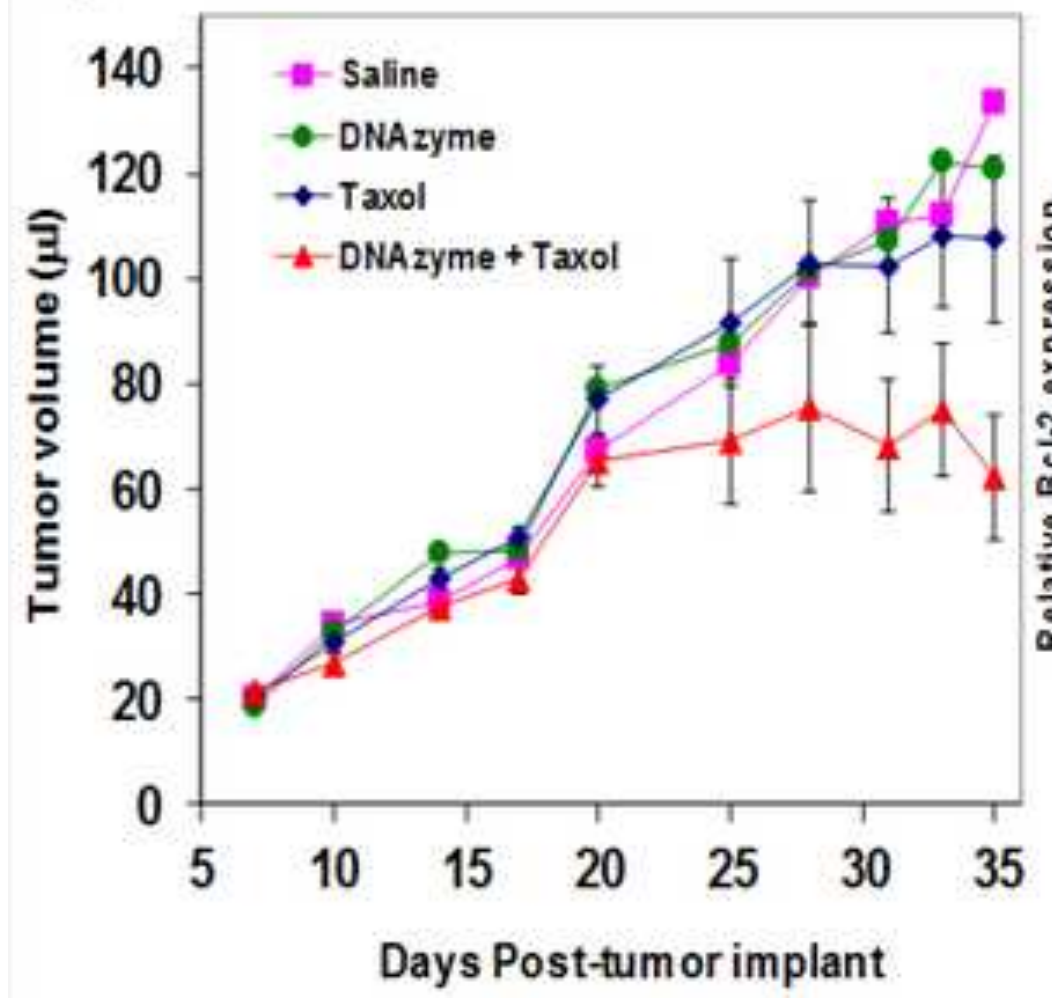

B

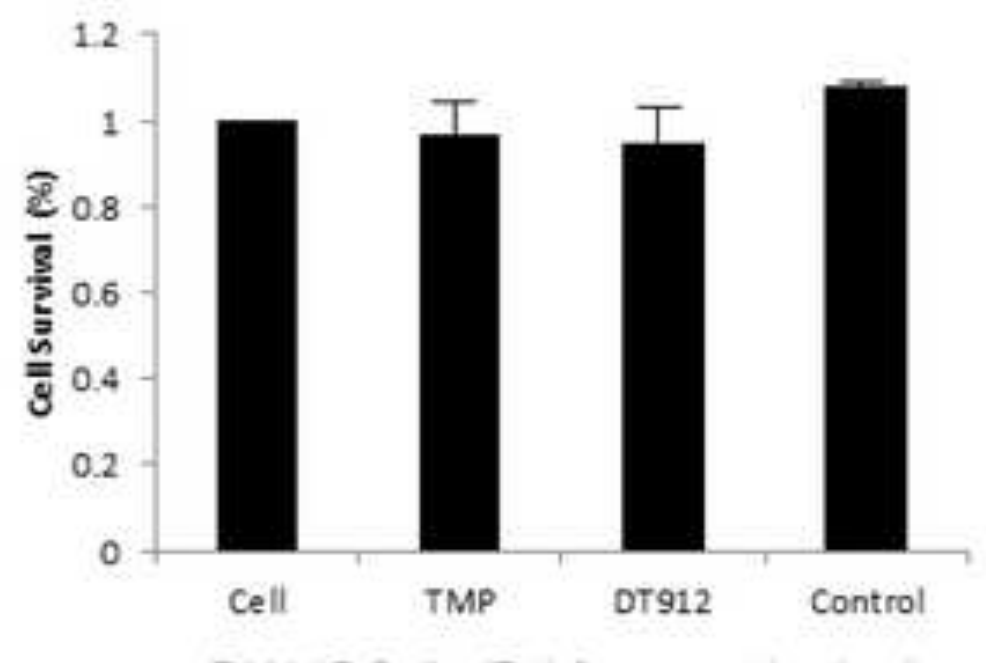

DU145 Cells (Bcl-2 expression low)

D

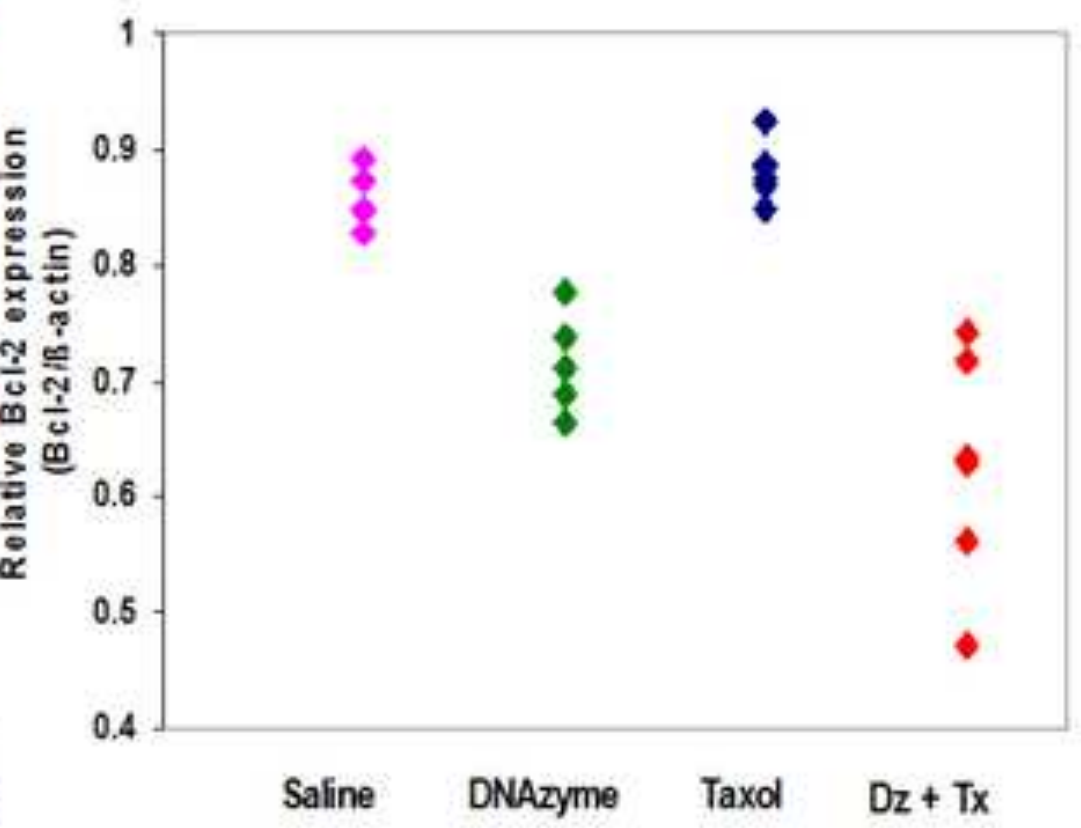

